Die Kunst der Utopie 
Wolfgang Braungart

\section{DIE KUNST DER UTOPIE}

Vom Späthumanismus zur früben Aufklärung

J.B. Metzlersche Verlagsbuchhandlung Stuttgart 
CIP-Titelaufnahme der Deutschen Bibliothek

Braungart, Wolfgang:

Die Kunst der Utopie : vom Späthumanismus zur frühen Aufklärung / Wolfgang Braungart. - Stuttgart : Metzler, 1989

ISBN 978-3-476-00658-5

ISBN 978-3-476-03277-5 (eBook)

DOI 10.1007/978-3-476-03277-5

Dieses Werk einschließlich aller seiner Teile ist urheberrechtlich geschützt. Jede Verwertung außerhalb der engen Grenzen des Urheberrechtsgesetzes ist ohne Zustimmung des Verlages unzulässig und strafbar. Das gilt insbesondere für Vervielfältigungen, Übersetzungen, Mikroverfilmungen und die Einspeicherung und Verarbeitung in elektronischen Systemen.

(C) 1989 Springer-Verlag GmbH Deutschland

Ursprünglich erschienen bei J.B. Metzlersche Verlagsbuchhandlung und Carl Ernst Poeschel Verlag GmbH in Stuttgart 1989 
Für Jutta 


\section{Vorbemerkung}

Seite 8

\section{Einleitung}

Seite 9

\section{Johann Valentin Andreae: \\ Reipublicae Christianopolitanae Descriptio (1619)}

Seite 16

1. Einleitung 16-2. Allegorie 21-3. Artefakt 44-4. Forschen und Arbeiten: Progressive Naturaneignung 54-5. Sammeln und Ordnen: Die Utopie als Kunstkammer 74-6. Erinnern und Erziehen: Kunst als Mittler zur Welt 77

\section{Utopische Alternativen}

Seite 82

1. Tommaso Campanella: La Città del Sole (1602/1623) 82

2. Francis Bacon: Nova Atlantis 92

\section{Die Utopie als Kunstkammer}

Seite 106

1. Samuel von Quicchebergs ideale Kunstkammer 106-2. Die Uffizien-Tribuna: gebaute Utopie 110 - 3. Francescos Studiolo: Mythos als Ordnungsverfahren 114 4. Integration der Wortwissenschaften, Anschluß an den Fürstenhof 120 5. Naturalienkabinette der Neugierde 125-6. Das Vorbild Salomons: Aneignung eines heilsgeschichtlichen Typus 128-7. Gesamtkunstwerke von Kunst, Wissenschaft und Technik 131-8. Ordnungsprobleme und Auszug der Kunst 136 
V. Johann Daniel Majors See $=$ fahrt nach der neuen Welt (1679)

Seite 148

1. Theoriedefizite und mißlingende Utopie $148-2$. Galantes Experimental-Studium als Legitimationskonzept der neuen Wissenschaften 158

\section{Ziglers Banise und Veiras' Histoire des Sévarambes}

Seite 170

1. Einleitung $170-2$. Der höfisch-historische Roman als Utopie-Ersatz: Ziglers Banise 176 - 3. Denis Veiras: Histoire des Sévarambes 181

\section{Philipp Balthasar Sinold:}

Die glückseeligste Insul auf der gantzen Welt

Seite 211

Einschränkung der Utopie im Zeichen des Pietismus 211

VIII. Johann Gottfried Schnabel:

Wunderliche Fata einiger See $=$ Fahrer

Seite 217

1. Einleitung: Die Romanvorrede $217-2$. Neugierde auf Geschichten $221-$ 3. Idealgeschichte der Menschheit 235 - 4. Magie: Ergänzung und Korrektur felsenburgischer Rationalität 247

\section{Perspektiven}

Seite 262

1. Universalgeschichte statt Universaltopik: Schillers Jenaer Antrittsvorlesung 263

2. Idealpädagogik und Universalwissenschaft als Karikatur: Schummels Spitzbart 265

3. Verständigungsprobleme: Wezels Robinson Krusoe 268

\section{$X$. Anhang}

Seite 273

1. Hinweise zur Schreib- und Zitierweise 273-2. Literaturverzeichnis 274

3. Abbildungen $315-4$. Register 339 


\section{VORBEMERKUNG}

Dies ist die leicht überarbeitete Fassung meiner Dissertation, die im Herbst 1986 unter dem Titel `Ordnung und Integration. Zum Wandel der frühneuzeitlichen Utopie ‘ vom Fachbereich für Philosophie und Sozialwissenschaften der Technischen Universität Braunschweig angenommen worden ist. Seither erschienene Literatur konnte ich in der Regel nur im Literaturverzeichnis berücksichtigen.

Es ist mir ein Bedürfnis, an dieser Stelle denen zu danken, die mir in den Jahren des Entstehens dieser Arbeit geholfen haben:

Zuerst möchte ich Prof. Dr. Jürgen Stenzel, Braunschweig, danken, der die Arbeit betreute und begutachtete, und dem Zweitgutachter, Prof. Dr. Gerhard Kurz, Gießen, als dessen Wissenschaftlicher Mitarbeiter seit 1985 ich die günstigen Arbeitsmöglichkeiten hatte, die mir die endgültige Ausarbeitung erlaubten. Beide haben diese Arbeit nicht nur in vielen Gesprächen mit zahlreichen Hinweisen und freundlicher Kritik unterstützt; sie haben sie auch (und das war nicht weniger wichtig) in den Phasen mit großem Vertrauen verteidigt, in denen ich selbst vielleicht nicht mehr so sehr vom Sinn und Ziel des Projektes überzeugt war.

Prof. Dr. Horst Bredekamp, Hamburg, hat mich 1980 in seinem Zürcher Seminar auf den kunstgeschichtlichen Zugang zum Thema hingewiesen, war auch in den folgenden Jahren stets zur Diskussion bereit und ist schließlich die gesamte Arbeit noch einmal kritisch durchgegangen. Prof. Henning Freiberg, Braunschweig, hat 1981/82 eine ausführliche Version des kunsthistorischen Kapitels als Examensarbeit ermöglicht. Prof. Dr. Wilhelm Kühlmann, Heidelberg, Dr. Christoph Jermann, Rom/Neapel, und mein Bruder, Dr. Georg Braungart, Tübingen, haben die Arbeit freundlich und kritisch gelesen und viele Verbesserungen angeregt. Dr. Martin Leutzsch, Bochum, hat die Übersetzungen aus dem Lateinischen durchgesehen. Ernst Golawski, Wolfsburg, hat unermüdlich bei den Korrekturarbeiten geholfen.

Ihnen allen danke ich herzlich wie auch den Damen und Herren der Universitätsbibliotheken Braunschweig und Gießen und der Herzog August Bibliothek Wolfenbüttel. Danken möchte ich auch Dr. Peter Scherber und Andrea Schäfer von der Gesellschaft für wissenschaftliche Datenverarbeitung, die mir halfen, das Typoskript in Computer einzulesen.

Schließlich danke ich auch der Deutschen Forschungsgemeinschaft für einen großzügigen Druckkostenzuschuß, der die Publikation der Arbeit in der vorliegenden Weise erst möglich gemacht hat.

Widmen aber möchte ich die Arbeit meiner Frau. Ohne ihre Bereitschaft zum Zuhören, zur Kritik, zur Diskussion, ohne ihre ausdauernde Hilfe beim Schreiben und Korrigieren hätte ich die Arbeit nicht in dieser Form abgeschlossen. 